[0212-7199 (2008) 25: 5; pp 205-208] ANALES DE MEDICINA INTERN Copyright (C) 2008 ARAN EDICIONES, S.L.

AN. MED INTERNA (Madrid) Vol. 25, N. ${ }^{\circ}$ 5, pp. 205-208, 2008

\title{
Detección y manejo inicial del síndrome de respuesta inflamatoria sistémica en las urgencias de medicina. Análisis de 24 horas en un hospital general
}

\author{
F. J. CANDEL, F. MARTÍNEZ-SAGASTI ${ }^{1}$, M. MATESANZZ2 , J. GONZÁLEZ DEL \\ CASTILLO $^{3}$, F. ORTUÑO ${ }^{1}$, F. J. MARTÍN ${ }^{3}$, A. MONEO ${ }^{1}$, I. CANDEL ${ }^{2}$
}

Servicios de Microbiología Clínica, ${ }^{1}$ Medicina Intensiva, ${ }^{2}$ Medicina Interna, ${ }^{3}$ Urgencias . Hospital Clínico San Carlos. Universidad Complutense. Madrid

\begin{abstract}
DETECTION AND INICIAL MANAGEMENT OF THE SYSTEMIC INFLAMMATORY RESPONSE SYNDROME IN MEDICINE EMERGENCY ROOM. 24 HOURS FOLLOW-UP IN A GENERAL HOSPITAL
\end{abstract}

\section{RESUMEN}

Introducción: El síndrome de respuesta inflamatoria sistémica (SIRS) se presenta con frecuencia en Urgencias. Desde la Conferencia de Consenso de 1991, ratificada en la Conferencia Internacional de definiciones de sepsis de 2001 se define este síndrome como la adaptación del organismo tras una agresión. Su detección precoz y la instauración de medidas de soporte, guiadas por objetivos, mejoran el pronóstico de los pacientes.

Objetivos: Conocer la incidencia de SIRS entre los pacientes que acuden a Urgencias, sus factores de frecuencia y distribución y su evolución clínica a los 3 y 30 días.

Pacientes y métodos: Estudio prospectivo observacional, simple ciego. Se detectó la situación de SIRS mediante observadores con registro independiente, y se observó su manejo, durante 24 horas. Se excluyeron los pacientes de obstetricia y traumatología.

Seguimiento: Telefónico y mediante la estación informática de pacientes a los 3 y 30 días. Análisis estadístico: descriptivo.

Resultados: De las 163 asistencias se encontraron 25 pacientes con SIRS $(15,3 \%), 16$ en varones $(65 \%)$ y 9 en mujeres (35\%). Por edades había $8<30$ años, 4 de 30-60 años y $13>60$. A 12 se los 25 casos de SIRS $(48 \%)$ no se les midió la frecuencia respiratoria. En 19 de los 25 casos $(76 \%)$ el origen fue infeccioso (sepsis). El parámetro definitorio de SIRS más frecuente fue la taquicardia seguido del trastorno leucocitario. Tan sólo se inició precozmente la fluidoterapia y la antibioterapia en el 21 y $42 \%$ respectivamente de las sepsis. Ingresaron 15 de los 25 (2 en UCI), 3 días después permanecían 11 ingresados y 30 días después 2.

Conclusiones: El SIRS es una situación clínica prevalente en Urgencias de medicina, mayoritariamente de etiología infecciosa (sepsis) que depara un número considerable de ingresos. Es necesaria una mayor sensibilización por parte del colectivo medico para establecer prontamente su diagnóstico e iniciar precozmente el tratamiento de soporte que mejore el pronóstico de estos pacientes.

PALABRAS CLAVE: SIRS. Sepsis. Shock séptico. Urgencias.

\section{ABSTRACT}

Introduction: Systemic Inflammatory Response Syndrome (SIRS) is a clinical situation frequently observed in Emergency Room (ER). Its early detection and supporting measures improve prognosis of these patients.

Aims: To know the incidence of SIRS among patients who come to $E R$, their frequency and distribution factors and the clinical evolution at 3 and 30 days.

Patients and methods: Observational prospective simple-blind study. During 24 hours, SIRS was detected by observant doctors with an independent registry. Their management was observed. Patients from Obstetrics and Traumatology were not observed. Follow-up was done using telephonic and informatical techniques at 3 and 30 day. A descriptive analysis was done.

Results: There were 163 patients attended in ER; 25 of them with SIRS $(15.3 \%), 16$ were male (65\%) and 9 female (35\%). By ages 8 were under 30, 4 were between 30 and 60 and 13 were over. The respiratory rate was not measured in 12 of the 25 patients with SIRS (48\%). An infectious etiology (sepsis) was found in 19 of those 25 (76\%) patients. The most frequent criterion of sepsis was tachycardia, followed by leukocyte disorders. Support of volume and antimicrobial therapy were only started at once in 21 and $42 \%$ respectively on patients with sepsis. 15 of 25 were admitted ( 2 in ICU). After 3 days, 11 of 25 remained at hospital and after 30 days 2 .

Conclusions: SIRS is a prevalent situation in ER with a high percentage of admissions. Most of SIRS were of infectious origin (sepsis). Major attention is needed among physicians to establish a promptly diagnose and starting support measures that improve their prognosis.

KEY WORDS: SIRS. Sepsis. Septic shock. Emergency room.

Candel FJ, Martínez-Sagasti F, Matesanz M, González del Castillo J, Ortuño F, Martín FJ, Moneo A, Candel I. Detección y manejo inicial del síndrome de respuesta inflamatoria sistémica en las urgencias de medicina. Análisis de 24 horas en un hospital general. An Med Interna (Madrid) 2008; 25: 205-208. 


\section{INTRODUCCIÓN}

El SIRS (del inglés Systemic Inflammatory Response Syndrome) es una situación clínica de respuesta inflamatoria general a una agresión, ya sea esta por una infección (sepsis), un traumatismo o una cirugía, aunque otras situaciones clínicas son también capaces de producirlo (TEP, ICC, etc.) $(1,2)$. Se detecta clínicamente por la presencia simultánea en un paciente de dos o más de entre los siguientes criterios: frecuencia cardiaca superior a $90 \mathrm{lpm}$, frecuencia respiratoria mayor de $20 \mathrm{rpm}$, temperatura axilar superior a $38{ }^{\circ} \mathrm{C}$ o inferior a $36{ }^{\circ} \mathrm{C}$, presencia de más de 12.000 leucocitos $/ \mathrm{mm}^{3}$ o menos de 4.000 leucocitos $/ \mathrm{mm}^{3}$ o la aparición de mas de un $10 \%$ de cayados en el recuento diferencial (3). La detección precoz de esta situación clínica y el subsiguiente inicio de medidas de soporte (hidratación, antimicrobianos, oxígeno, etc.) es capaz de reducir la morbimortalidad en estos pacientes.

Con el objeto de conocer la incidencia de SIRS, sus factores de frecuencia y distribución, el impacto que este síndrome supone en el manejo diagnóstico y terapéutico del personal sanitario y la evolución clínica de estos pacientes a los 3 y 30 días de su asistencia en el Servicio de Urgencias, se planteó un estudio observacional en el Hospital Clínico Universitario San Carlos de Madrid.

\section{MATERIAL Y MÉTODOS}

Se trata de un estudio observacional prospectivo y simple ciego en el que se detectaba la situación de SIRS, así como sus parámetros clinicoepidemiológicos por médicos de Urgencias que permanecían como observadores junto al médico adjunto de guardia y que cumplimentaban un registro independiente, sin informar a los clínicos responsables del paciente. A continuación se estableció un seguimiento a los días 3 y 30 . El tipo de muestreo fue de casos consecutivos, la ubicación el Ârea de Urgencias de Medicina (Unidad de Agudos y Unidad de Primera Asistencia) del Servicio de Urgencias del Hospital Clínico Universitario San Carlos de Madrid. El tiempo de observación fue de 24 horas (de forma ininterrumpida desde las 8:00 a.m. de 2/3/06 hasta las 8:00 a.m. de 3/3/06). El sistema de Registro fue un protocolo Clínico-Analítico en el que se anotaban datos demográficos, los parámetros definitorios de SIRS según los consensos internacionales (3), el diagnóstico en urgencias, el tratamiento de soporte o especifico que recibió el paciente y la hora a la que lo recibió y donde se ubicó al paciente. Posteriormente se realizó un seguimiento telefónico y a través de la Estación Informática de nuestro hospital (CLINICA). Se practicó un análisis descriptivo empleando valores absolutos y porcentajes.

\section{RESULTADOS}

De las 163 asistencias realizadas en el periodo de observación se encontraron un total de 25 pacientes con SIRS $(15,3 \%), 16$ varones $(65 \%)$ y 9 mujeres $(35 \%)$. A 12 de los 25 casos de SIRS (48\%) no se les midió la frecuencia respiratoria. La distribución por grupos etarios fue la siguiente: había 8 pacientes menores de 30 años, 4 de entre 30 y 60 años y 13 mayores de 60 . Cinco de los pacientes tenían como antece- dente una neoplasia, 2 eran diabéticos y en 10 subyacía la disfunción de un órgano. En 19 de los 25 casos el origen fue infeccioso (sepsis). Once consultaron por síntomas respiratorios, 6 por urinarios, 3 por patología abdominal y 5 por otros motivos (ORL, Neurología). El criterio definitorio de SIRS más frecuente fue la taquicardia, seguido del trastorno leucocitario. La mayoría de los casos de SIRS detectados, 19 (76\%) de los 25, tenían como causa una infección (sepsis). Tan sólo se inició precozmente la fluidoterapia y la antibioterapia en el 21 y $42 \%$ respectivamente de las sepsis. Se solicitaron estudios complementarios a Microbiología en el $83 \%$ de las situaciones clínicas con indicación de estos. Ingresaron 15 de los 25 ( 2 en UCI), 3 días después permanecían 11 ingresados y a los 30 días sólo 2 . Hubo un fallecimiento en un paciente con un ACV. De entre las altas, 2 pacientes acudieron de nuevo al Servicio de Urgencias por el mismo motivo de consulta en el mes subsiguiente (Tablas I-III y Figuras 1-3).

\section{TABLA I}

\begin{tabular}{ll}
\multicolumn{2}{c}{ DESCRIPTIVOS INICIALES } \\
\hline Antecedentes SIRS & $N^{\circ}$ pacientes $25(\%)$ \\
\hline Varones & $16(65 \%)$ \\
Mujeres & $9(35 \%)$ \\
$<30$ años & $8(32 \%)$ \\
$30-60$ años & $4(16 \%)$ \\
$>30$ años & $13(52 \%)$ \\
Diabetes mellitus & $2(8 \%)$ \\
Insuficiencia renal crónica & $1(4 \%)$ \\
HTA & $6(24 \%)$ \\
Insuficiencia hepática & $2(8 \%)$ \\
Insuficiencia respiratoria & $5(20 \%)$ \\
Insuficiencia cardiaca & $1(4 \%)$ \\
Neoplasia & $2(8 \%)$ \\
Tratamiento esteroideo & $5(20 \%)$ \\
\hline
\end{tabular}

\section{TABLA II}

MOTIVO DE CONSULTA EN LOS PACIENTES CON SIRS

\begin{tabular}{lcc}
\hline Motivo de consulta en pacientes con SIRS & $\begin{array}{c}\text { Total } \\
\text { pacientes }\end{array}$ & $\begin{array}{c}\text { Infecciosos } \\
\text { (sepsis) }\end{array}$ \\
\hline $\begin{array}{l}\text { Síntomas respiratorios (ICC, TEP, NAC) } \\
\text { Síntomas urinarios (CRU, ITU, PNA) }\end{array}$ & 11 & 7 \\
$\begin{array}{l}\text { Sintomas abdominales (dolor FID, } \\
\quad \text { colecistitis, diarrea) }\end{array}$ & 6 & 5 \\
$\begin{array}{l}\text { Otros (ACV, sinusitis, viriasis, infección } \\
\text { pie diabético) }\end{array}$ & 3 & 3 \\
$\begin{array}{l}\text { Total } \\
\text { Tol }\end{array}$ & 5 & 4 \\
\end{tabular}

ICC: insuficiencia cardiaca congestiva; TEP: tromboembolismo pulmonar; NAC: neumonía adquirida en la comunidad; CRU: crisis renoureteral; ITU: infección del tracto urinario; PNA: pielonefritis aguda; FID: fosa iliaca derecha; ACV: accidente cerebrovascular.

\section{DISCUSIÓN}

Si bien el SIRS puede obedecer a una cirugía, un traumatismo, una quemadura o a fenómenos cardiopulmonares vasculares (ICC, TEP), la causa más frecuente comunicada en la 
TABLA III

\begin{tabular}{|c|c|c|c|c|c|c|c|}
\hline \multicolumn{8}{|c|}{ TABLA III } \\
\hline \multicolumn{8}{|c|}{ EVOLUCIÓN DEL SIRS EN LOS PACIENTES ESTUDIADOS } \\
\hline & $n=25$ & & $1^{\text {er }}$ día & & $3^{e r}$ día & & $30^{\circ}$ día \\
\hline \multirow[t]{5}{*}{ Ingresos } & & Sala B/UCE & 5 & Sala B/UCE & - & Sala B/UCE & - \\
\hline & & Planta* & 7 & Planta* & 9 & Planta* & 2 \\
\hline & & Cirugía & 1 & Cirugía & 1 & Cirugía & - \\
\hline & & $\mathrm{UCl}$ & 2 & $\mathrm{UCl}$ & - & $\mathrm{UCl}$ & - \\
\hline & 15 & Exitus & - & Exitus & 1 & Exitus & - \\
\hline \multirow[t]{2}{*}{ Altas } & & & & $\begin{array}{l}\text { Acude por el } \\
\text { mismo motivo }\end{array}$ & 1 & $\begin{array}{l}\text { Acude por el } \\
\text { mismo motivo }\end{array}$ & 1 \\
\hline & 10 & & & Exitus & - & Exitus & - \\
\hline
\end{tabular}

Planta* incluye planta de MIN, planta de hospital de apoyo, planta de especialidades y unidad de hospitalización a domicilio.

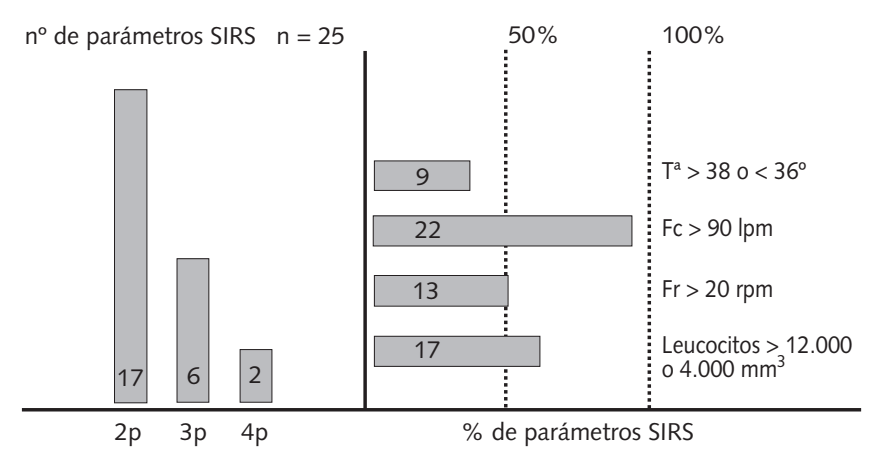

Fig 1. Incidencia de parámetros definitorios de SIRS en la muestra estudiada.

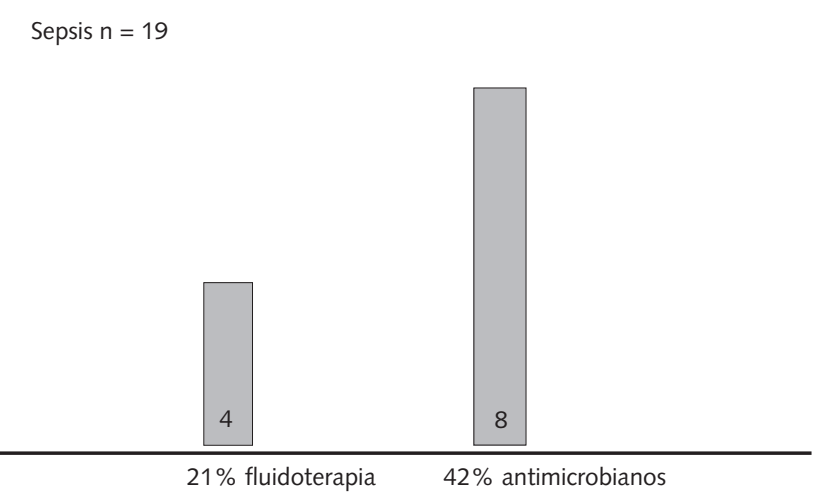

Fig 2. Tratamiento con fluidoterapia y antimicrobianos en las primeras 4 horas de permanencia en urgencias (sepsis).

literatura médica es la infección (sepsis) (1). Precisamente se buscaron unos criterios muy sensibles aunque poco específicos para que pudieran detectarse pronto los paciente sépticos. La sepsis es un fenómeno clínico que aparece con una frecuencia que oscila en torno al $10 \%$ en los Servicios de Urgencias $(4,5)$ y que a menudo es infravalorado. Uno de los motivos de su infravaloración puede ser el hecho de que una medida tan fácil de la exploración inicial del paciente como es

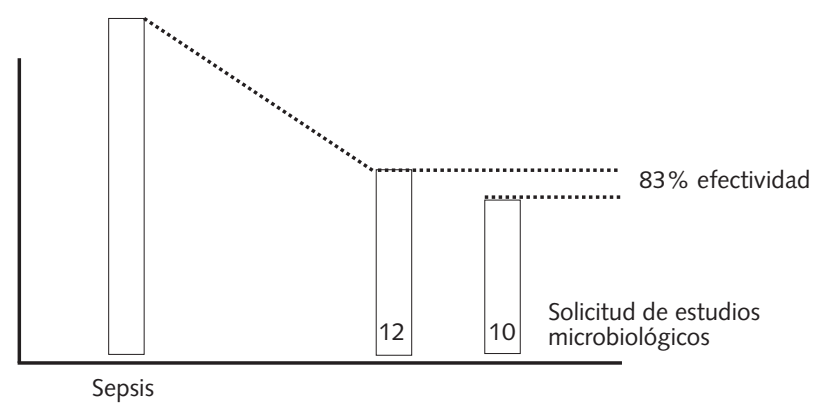

Se solicitaron 10 estudios a microbiología: 3 hemocultivos, 5 urocultivos, 1 esputo y 1 exudado.

Fig 3. Situaciones con indicación clínica de estudio microbiológico para dirigir el tratamiento.

la toma de la frecuencia respiratoria se hace sólo en la mitad de los casos, resultando curioso que se solicita una analítica antes que lo que se realiza la toma de la frecuencia respiratoria.

Un tercio de las sepsis en los Servicios de Urgencias se terminan transformando en sepsis grave y un $9 \%$ en shock séptico (5). La sepsis grave es aquella situación de sepsis caracterizada por la disfunción de al menos un órgano (función hemodinámica, renal, respiratoria, hepática, hematológica o neurológica), o por la mala perfusión tisular (hiperlactacidemia), que requiere expansores plasmáticos para su corrección. El shock séptico se caracteriza por la presencia de hipotensión arterial que no responde a expansión del volumen intravascular y requiere soporte de aminas vasoactivas (3). La mortalidad de la sepsis en nuestro medio se estima en torno a 97 casos por 100.000 habitantes año. Según datos del estudio EDU-SEPSIS6 a finales de 2005, en España la sepsis grave presentó una mortalidad del $47 \%$, y el shock séptico del $84 \%$, superando ampliamente a la mortalidad acaecida por el infarto de miocardio o el ictus.

El conocimiento del impacto en la morbimortalidad tan importante que la sepsis presenta en la población ha suscitado el interés por las principales sociedades médicas, que se ha traducido en la elaboración de documentos de consenso para 
detectar el proceso y establecer precozmente las medidas de soporte y el tratamiento antimicrobiano empírico (7). Está demostrado que la administración precoz de líquidos (por ejemplo 20-30 ml/kg en media hora, salvo contraindicación por gestación, quimioterapia, cardiopatía estructural, etc.) con la intención de restablecer la situación hemodinámica, mejora la supervivencia (8). Del mismo modo se conoce la influencia del retraso en la administración de antimicrobianos en la mortalidad. Así, según se administre el antimicrobiano en 30 minutos o en las primeras 9-12 horas, la mortalidad varía entre un 17 y un $74 \%$ (9).

En el año 2002 surgió un movimiento de concienciación respaldado por la European Society of Intensive Care Medicine, el International Sepsis Forum, la Society of Critical Care Medicine y el American College of Emergency Physicians, llamado Surviving Sepsis Campaign o "Campaña para sobrevivir a la sepsis" (CSS) (10). Este movimiento, que cada vez goza de mayor aceptación y seguimiento por intensivistas, emergenciólogos e internistas del mundo entero, se trazó como objetivo la reducción de la mortalidad en un $25 \%$ en 5 años (2005-2009). El citado documento establecía una serie de recomendaciones y objetivos, entre los que destacamos: la identificación precoz del cuadro séptico mediante la clínica y los parámetros de SIRS, el comienzo de la fluidoterapia (30 ml/kg en menos de una hora), la realización de analítica con lactato, la toma de hemocultivos, la obtención de una saturación venosa central superior al 70\%, la administración de la primera dosis de antibiótico adecuado y el aviso al intensivista en menos de 2 horas, independientemente de la ubicación inicial del enfermo.

Aunque la sepsis y el shock séptico suponen un reto terapéutico (11), algunas medidas sencillas como la administración precoz de fluidos para restablecer la presión de perfusión han demostrado su eficacia y no deben retrasarse. En nuestro corte observacional constatamos una demora significativa en el inicio de la fluidoterapia y el tratamiento antimicrobiano empírico (Fig. 2). Sin embargo llama la atención la excelente efectividad con la que los médicos de urgencias solicitaron los estudios microbiológicos complementarios para esclarecer el foco infeccioso (Fig. 3). Quizá nuestros médicos de urgencias no estén suficientemente concienzados de la importancia del soporte hemodinámico inicial en la sepsis y las órdenes de prescripción no se llevan a cabo en el acto, sino que se adecúan a un horario posológico en las hojas de enfermería, quizá la presión asistencial creciente reduce a intenciones los actos. En cualquier caso es importante concienciarse de que la sepsis es una situación inflamatoria dinámica con un pronóstico tiempo-dependiente, comparable en trascendencia clínica a la cardiopatía isquemia o a la enfermedad cerebrovascular, y que, del mismo modo que existe en los hospitales la figura del "cardiólogo de guardia de coronarias" para el manejo del síndrome coronario agudo o el "neurólogo de guardia" para afrontar el Código Ictus, convendría disponer de un sepsis team multidisciplinar (integrado por intensivistas, radiólogos, cirujanos, microbiólogos, internistas o emergenciólogos), con capacidad crítica para implementar las recomendaciones de los consensos (soporte hemodinámico inicial, antibioterapia y control del foco).

Llama por último la atención el alto índice de ingresos que el SIRS conlleva, que ascendió a 15 de 25 pacientes $(60 \%)$ en las primeras 24 horas, que a las 72 horas todavía era de 11 pacientes (44\%). Un mes después aún quedaban 2 pacientes con SIRS ingresados.

Como conclusión y en base a los resultados obtenidos con nuestra muestra estudiada, entendemos que el SIRS es una situación clínica prevalente en los servicios de urgencia hospitalarios que depara un número considerable de ingresos. También que la mayoría de los SIRS en el Área Médica de Urgencias son de origen infeccioso (sepsis) y, por ultimo, que es necesaria una mayor sensibilización por parte del colectivo médico para detectar a estos pacientes tomando la temperatura, la frecuencia cardiaca y la respiratoria sistemáticamente a todos los pacientes que se atiendan en el área de urgencias y poder establecer el diagnóstico de SIRS, buscar la causa e iniciar precozmente el tratamiento de soporte que mejore el pronóstico de estos pacientes.

\section{Bibliografía}

1. Brun-Buisson, C. The epidemiology of the systemic inflamatory response. Intensive Care Med 2000; 26 (Supl. 1): S64-S74.

2. Muckart DJ, Bhaguanjee S. American College of Chest Physicians/Society of critical Care Medicine Consensus Conference Definitions of the Systemic Inflammatory Response Syndrome and Allied Disoreders in Relation to Critically Injured Patients. Crit Care Med 1997; 25: 1789-1795.

3. Levy MM, Fink M P, Marshall JC, Abraham E, Angus D, Cook D, et al. 2001 SCCM/ESICM/ACCP/ATS/SIS International Sepsis Definitions Conference. Intensive Care Med 2003; 29: 530-538.

4. Grupo para el Estudio de la Infección en Urgencias. Estudio epidemiológico de las infecciones en el área de urgencias. Emergencias 2000; 12: 80-89.

5. Esteban A, Frutos-Vivar F, Ferguson ND, Gordo F, Honrubia T, Penuelas $\mathrm{O}$, et al. Incidence and outcome of sepsis in a health area from Madrid, Spain. 100th Internacional Conference ATS, Orlando 2004.

6. Impacto de un programa educacional basado en la campaña sobrevivir a la sepsis en España http://www.edusepsis.semicyuc.org/

7. Documento de Consenso (SEMES-SEMICYUC). Recomendaciones del manejo diagnóstico-terapéutico inicial y multidisciplinario de la sepsis

grave en los Servicios de Urgencias hospitalarios: León Gil C (coordinación); García-Castrillo Riesgo L (coordinación); Moya Mir M (revisión); Artigas Raventós A (revisión), Borges Sa M, Candel González FJ, Chanovas Borrás M, Ferrer Roca R, Julián Jiménez A, Loza Vázquez A, Sánchez García M (miembros del Grupo de Expertos). Med Intensiva 2007; 31: 375-38.

8. Rivers E, Nguyen B, Havstad S, Ressler J, Muzzin A, Knoblich B, et al. Early goal-directed therapy in the treatment of severe sepsis and septic shock. N Engl J Med 2001; 345: 1368-1377.

9. Kumar A, Roberts D, Wood KE, Light B, Parrillo JE, Sharma S, et al. Duration of hypotension before initiation of effective antimicrobial therapy is the critical determinant of survival in human septic shock. Crit Care Med 2006; 34:1589-1596.

10. Dellinger RP, Carlet JM, Masur H, Gerlach H, Calandra T, Cohen J, et al. Surviving Sepsis Campaign guidelines for management of severe sepsis and septic shock. Crit Care Med 2004; 32: 858-873.

11. Durán Giménez-Rico HJ, Aller Reyero MA, Lorente Ruigómez L, Durán Giménez-Rico L, Arias Pérez J, Durán Sacristán H. Sepsis y shock séptico: un torbellino de mediadores inflamatorios de difícil manejo terapéutico. An Med Interna (Madrid) 2002; 19: 35-43. 